\title{
Introducción al estudio de la alteración de la semilla de girasol durante su almacenamiento
}

\author{
Por I. Otero Mateos, J.L. Casimiro-Soriguer Escofet, F. Romero Guzmán y J. Pereda Marín \\ Aceites y Proteínas, S.A. Apdo. Correos 1015 - 41080 Sevilla. \\ Dpto. Ingeniería Química. Facultad de Química. C/. Profesor García González s/n - 41012 Sevilla.
}

\section{RESUMEN}

Introducción al estudio de la alteración de la semilla de girasol durante su almacenamiento.

Se ha estudiado comparativamente la evolución de la alteración entre almacenamiento con atmósfera de aire y con corriente de nitrógeno, empleando semilla de dos tipos, para estudiar igualmente la influencia de su humedad: semilla ucraniana, con humedad en torno al $5 \%$, y semilla española (zona centro), con humedad superior al $12 \%$. En el caso de semilla ucraniana, el almacenamiento se ha realizado en cámara del $50 \%$ de humedad y $20^{\circ} \mathrm{C}$ para el caso del aire y en laboratorio para el almacenamiento con corriente de nitrógeno. La semilla de alta humedad ha sido conservada en el laboratorio, tanto para ambiente de aire como para la corriente de nitrógeno. A las muestras de semilla, tomadas periódicamente, se les realizaron análisis de humedad, mientras que al aceite crudo extraído de ellas se les realizaron análisis de grado de acidez, índice de $p$-anisidina, $k_{270}$, $k_{232}$ y contenido en compuestos polares.

PALABRAS-CLAVE: Aceite de girasol - Almacenamiento - Semilla de girasol (alteración).

\section{SUMMARY}

Introduction to the study of the changes in sunflower seeds during their storage.

A comparative study has been performed to examine the alterations occurring in sunflower seeds during their storage in an air atmosphere and in a nitrogen current. Two kinds of seeds with different moisture contents were used to examine the influence of this parameter: seeds from the Ukraine with about 5\% humidity, and Spanish seeds (from the Central region), with about $12 \%$ humidity. The Ukrainian seeds stored in an air atmosphere were kept at $20^{\circ} \mathrm{C}$ in a room with about $50 \%$ humidity, while those stored in a nitrogen current were kept in the laboratory. The seeds with a high moisture content were stored in the laboratory irrespective of the storage atmosphere used. Samples of seeds were taken periodically, and their moisture content was analyzed as was the acidity, $p$-anisidine, $k_{270}$ and $k_{232}$ values and \% polar compounds of their crude oil.

KEY-WORDS: Storage - Sunflower oil - Sunflower seed (alteration).

\section{INTRODUCCION}

La semilla de girasol presenta como principal problema su almacenamiento, ya que durante el mismo el aceite contenido en ella sufre alteraciones, observadas en datos industriales, que se deben al equilibrio entre la humedad del grano y la humedad relativa del aire intergranular como factor determinante (1-6). Este problema no se suele presentar en la semilla española del sur del país, pero sí es usual en las procedentes de la zona centro y norte de España, debido a que la campaña de girasol en estas zonas se dilata hasta finales de año, con el consiguiente aumento de su humedad debido a las lluvias. Además de la pérdida de calidad del aceite obtenido, existe un grave problema de seguridad, ya que en el almacenamiento de semilla húmeda se detectan calentamientos locales que pueden llegar a provocar la calcinación del grano, con las consiguientes pérdidas económicas, al no poder ser em pleado en ningún caso.

La semilla de girasol es un material higroscópico (7), siendo esta característica más acusada en el caso de granos biológicamente dañados por hongos e insectos (4). La aparición de este tipo de plagas se relaciona directamente con el aumento de humedad; por otra parte, ambos factores están interrelacionados con un aumento en el contenido de ácidos grasos libres del aceite crudo obtenido $(3,4,5,7,8,9)$. Durante el almacenamiento de la semilla de girasol, el proceso de hidrólisis se produce debido a su propia humedad (9), a las condiciones ambientales de humedad y temperatura $(8,9)$, a la concentración de la humedad en los espacios intergranulares $(1,2)$ y por último a la acción de hongos y plagas.

La humedad óptima del girasol está en torno al $7 \%$ para una temperatura de $20^{\circ} \mathrm{C}(9)$, ya que en estas condiciones se observa que el contenido en ácidos grasos libres se mantiene constante, a la vez que decrece la población de hongos.

Otro factor influyente en la evolución de la semilla es la temperatura, aunque en menor medida que la humedad (10). Se observa que a menor temperatura, la humedad tiende a mantenerse, mientras que al subir la temperatura presenta tendencia a aumentar.

La alteración de las semillas oleaginosas rotas se favorece en general debido a que de ese modo se facilita el ataque de microorganismos, la alteración enzimática, y la oxidación del aceite contenido en la semilla. Por otra parte, las semillas descascarilladas se conservan peor que las enteras. Este efecto de alteración por rotura se intensifica si se producen finos en lugar de cortes limpios.

Para inhibir en cierto modo la alteración, se han realizado estudios de conservación en atmósferas inertes a efectos de oxidación, usando principalmente nitrógeno y $\mathrm{CO}_{2}(10,11)$. Como conclusiones, se obtiene que resulta 
ventajoso el uso de estas atmósferas modificadas, ya que se consigue un estacionamiento de las variables estudiadas, principalmente de la acidez, con poco coste de instalación y mantenimiento, además de resultar económico desde el punto de vista energético comparado con los tratamientos de frío alternativos.

Por todo ello, el objetivo del presente trabajo es el estudio de la alteración global del aceite, medida a partir del grado de acidez, índice de $\mathrm{p}$-anisidina, $\mathrm{k}_{270}, \mathrm{k}_{232}$, y contenido global en compuestos polares, sin especificar la composición de éstos, que será objeto de un futuro trabajo.

\section{PARTE EXPERIMENTAL}

\subsection{Muestras}

Se usaron tubos como los de la fig. 1 para hacer el almacenamiento, en los que se introdujeron $50 \mathrm{~kg}$ de semilla en cada uno. Se empleó semilla de dos tipos:

- semilla ucraniana, con humedad en torno al $5 \%$

- semilla de producción nacional, procedente de la zona centro, con humedad del $12,69 \%$.

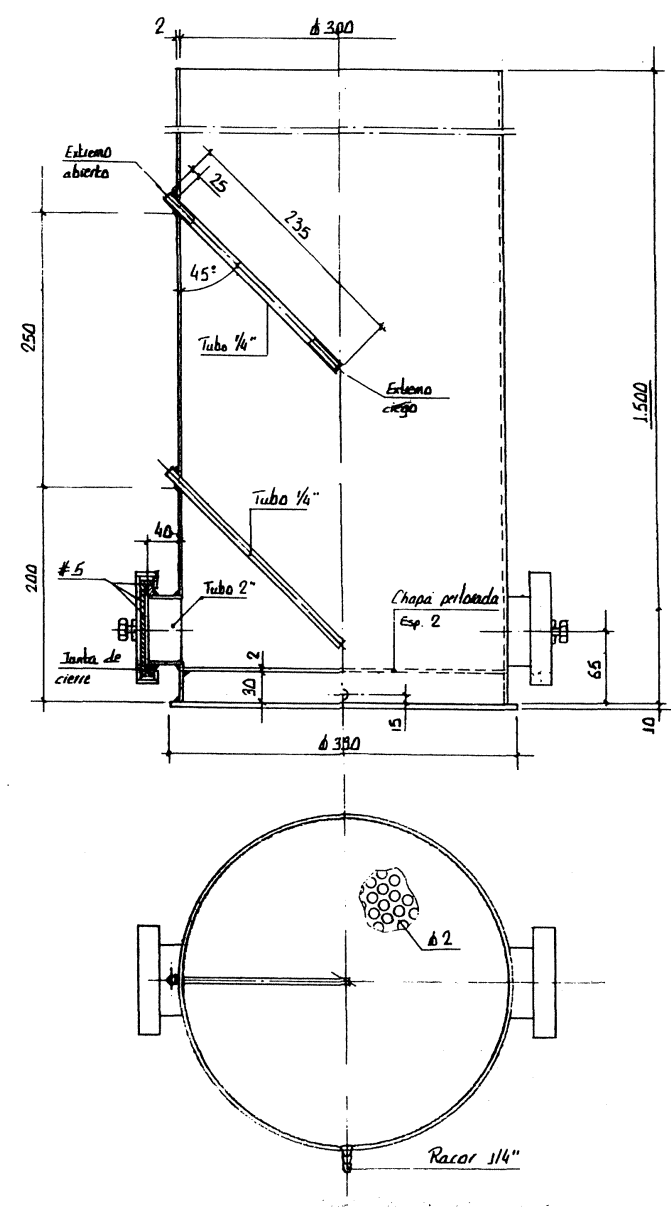

Figura 1
Con la semilla ucraniana se hicieron dos pruebas:

- almacenamiento en cámara con ambiente de aire en condiciones controladas ( $50 \%$ humedad y $20^{\circ} \mathrm{C}$ ).

- almacenamiento en laboratorio con corriente de nitrógeno de $50 \mathrm{ml} / \mathrm{min}$.

Por su parte, la semilla nacional, con humedad del $12,69 \%$, se almacenó en laboratorio, comparando la evolución de sus parámetros en atmósfera de aire y con corriente de nitrógeno de $50 \mathrm{ml} / \mathrm{min}$.

La elección de las condiciones de la cámara se hizo tomando la intermedia entre las cámaras disponibles, mientras que el flujo de nitrógeno se eligió en función del mínimo medible en el aparato del que se disponía, un medidor de flujo Chrompack, ya que se empleó nitrógeno de calidad para cromatografía.

La toma de muestras se realizó quincenalmente extrayendo por una de las bocas inferiores $1 \mathrm{~kg}$ de semilla aproximadamente, que era extractada mediante maceración en frío con hexano, para evitar en lo posible la degradación por calentamiento. Previamente a la extracción en frío se realizaron pruebas con el grado de acidez, observando que un aceite extractado en caliente por el método Soxhlet tiene un grado de acidez del orden de $0,3 \%$ superior al mismo aceite extractado en frío. La elección del disolvente se realizó en base a datos bibliográficos $(10,12)$ y a la mayor facilidad para obtenerlo, por ser el disolvente usado a nivel industrial.

\subsection{Análisis de las muestras}

A la semilla se le midió su contenido en humedad (13), y al aceite se le realizaron análisis de grado de acidez (14), absorbancia en el ultravioleta a 232 y $270 \mathrm{~nm}$ (medida de dienos y trienos conjugados, respectivamente) (15), índice de p-anisidina (medida de compuestos del tipo 2alquenal, formados en la etapa de terminación de la oxidación) (16), \% de compuestos polares (medida de la alteración global del aceite, es decir, glicéridos oxidados, dímeros y polímeros, monómeros cíclicos, y ácidos grasos libres) (17).

\section{DISCUSION}

En la gráfica 1 se presentan los datos correspondientes al grado de acidez, expresado como \% de ácido oleico, de la semilla ucraniana que contenía un $5 \%$ de humedad. Se observa que, en la semilla conservada en aire, el aumento de la humedad es constante y más rápido que en semilla conservada en nitrógeno. Por otra parte, no parece existir influencia del peso.

En la gráfica 2 se presentan los datos de evolución del grado de acidez de la semilla nacional con alto contenido de humedad. En este caso, la variación de este parámetro es más acusada que en el caso de semilla seca, pudiendo observar cómo en corriente de nitrógeno existe una permanencia de la acidez en valores aproximadamente constantes y cercanos a la unidad, mientras que en la semilla conservada en ambiente de aire existe un aumento brusco 


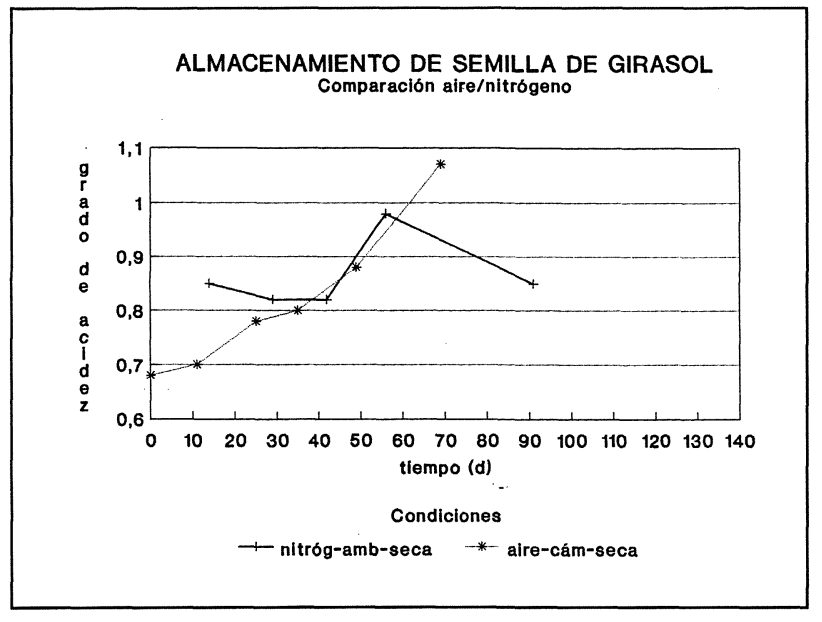

Gráfica 1

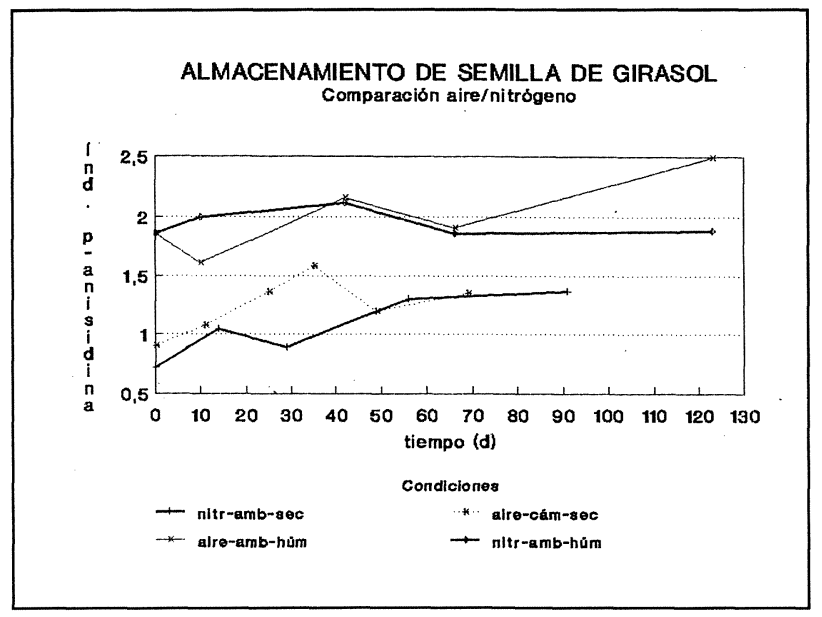

Gráfica 3

del cambio de acidez durante los primeros días de almacenamiento.

En la gráfica 3 se representan los datos correspondientes al índice de p-anisidina. No se puede observar grandes diferencias en los comportamientos en función de que haya atmósfera de aire o corriente de nitrógeno, aunque en el caso de semilla de alta humedad parece apreciarse una mayor tendencia al aumento en el caso de atmósfera de aire, sin poder obsenvar diferencias en función de la existencia de peso adicional para las otras muestras.

Los datos correspondientes al $k_{270}$ están representados en la gráfica 4 , pudiendo apreciar que la semilla húmeda presenta mayor constancia de valores que la semilla seca, aunque en ambos casos la corriente de nitrógeno permite que el crecimiento sea menor. Esto está explicado por el hecho de que el nitrógeno inhibe las reacciones de oxidación, efecto que también se puede apreciar en la gráfica 5 , correspondiente a los datos de coeficientes de extinción en el UV a $232 \mathrm{~nm}$ pero sólo para la semilla de poca humedad, aunque de forma menos acusada y más irregular; para la semilla húmeda no se puede observar influencia de la

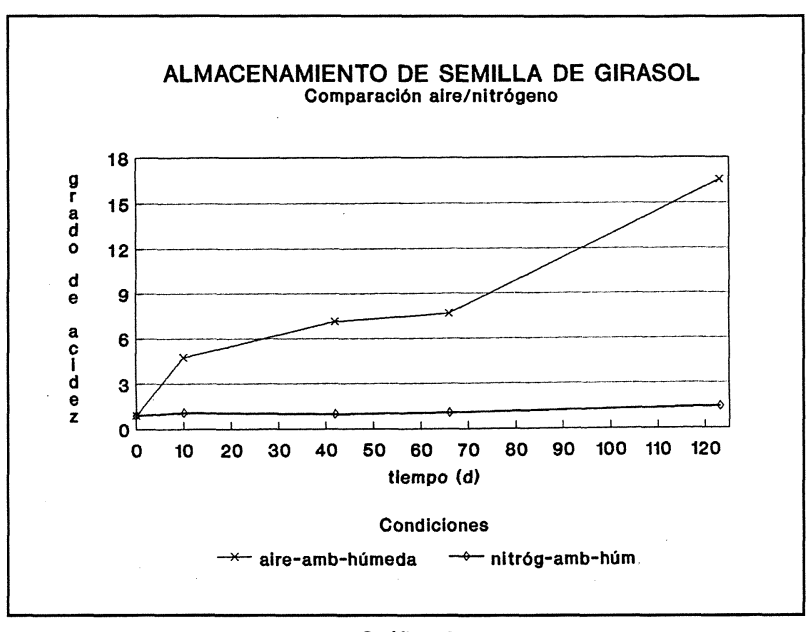

Gráfica 2

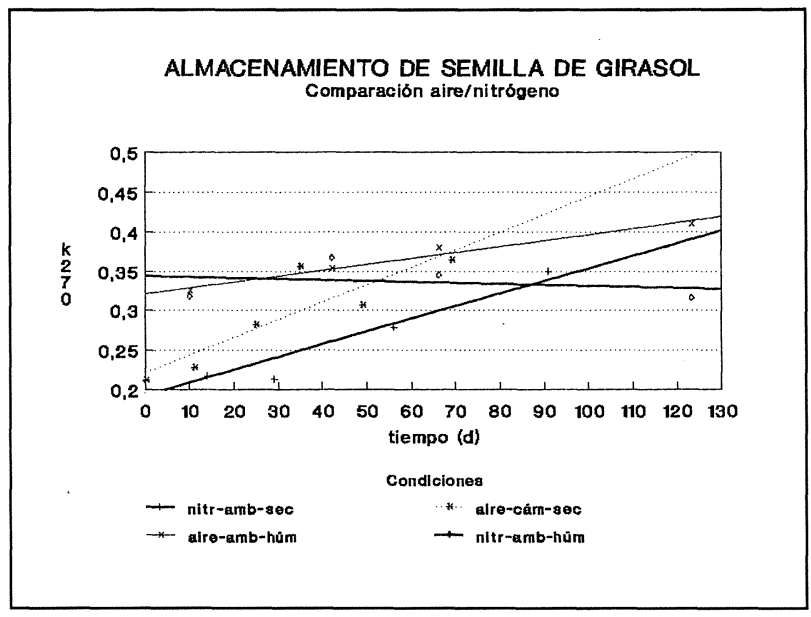

Gráfica 4

corriente de nitrógeno en el almacenamiento. Al igual que ocurría en el caso anterior, en la semilla de alta humedad se observa que los valores de este coeficiente se mantienen más constantes que los de la semilla seca. El hecho de que tanto el $k_{270}$ como el $k_{232}$ presenten tendencia a subir en el caso de la semilla con baja humedad, puede explicarse en base a que la deshidratación provoca un aumento de los compuestos de conjugación del aceite (18), además de que este tipo de compuestos son autocatalíticos, lo que favorecería la formación de nuevos dienos y trienos conjugados. Del mismo modo que en los otros parámetros estudiados, tampoco aquí se puede concluir nada de la influencia de la variable peso, tanto en el $k_{270}$ como en el $k_{232}$.

En la gráfica 6 se representan los valores de contenido en compuestos polares para la semilla de alta humedad. Se observa claramente que en el caso de la semilla conservada con atmósfera de aire se produce un crecimiento mucho más brusco en los valores de los compuestos de alteración que en la semilla almacenada con corriente de nitrógeno. La explicación a este hecho está en que con el nitrógeno se inhiben las reacciones de alteración, tanto 


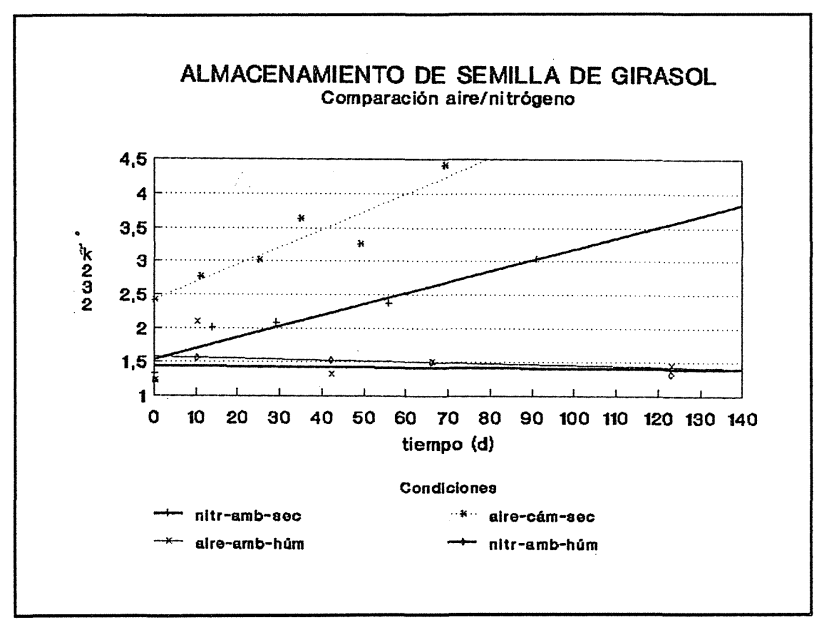

Gráfica 5

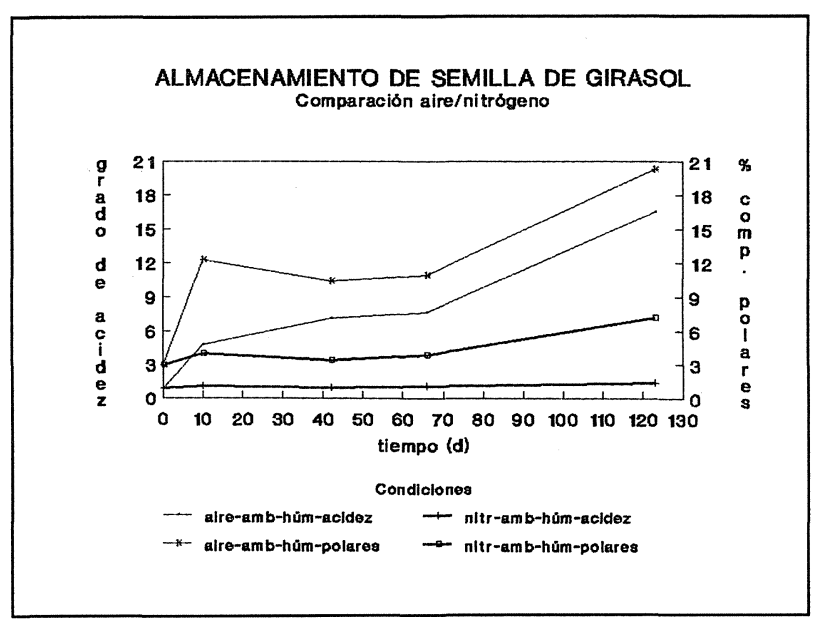

Gráfica 7

hidrolíticas, debido a la humedad del aire, que entra en equilibrio con la de la semilla, como oxidativas. Para observar mejor este efecto, en la gráfica 7 se representan conjuntamente en un diagrama de doble ordenada los valores de grado de acidez y de contenido en compuestos polares. Se puede apreciar una evolución muy similar de ambas líneas en el caso de la semilla húmeda, lo que indica que no se ha producido un aumento significativo de los compuestos de alteración originados por reacciones de tipo térmico y oxidativo, o al menos no son de importancia frente a las de tipo hidrolítico. En la semilla almacenada con corriente de nitrógeno, al principio existe una correspondencia en la evolución de ambas líneas, pero al final se produce un aumento en los compuestos polares con relación al grado de acidez; esto pudo deberse a un fallo en la conducción de nitrógeno, de modo que no le llegara a la semilla cantidad suficiente de inerte como para evitar que se produjeran procesos oxidativos.

En la gráfica 8 se representan los datos de contenido en humedad de la semilla, y salvo un punto que se sale claramente de la tendencia general para la muestra con alta

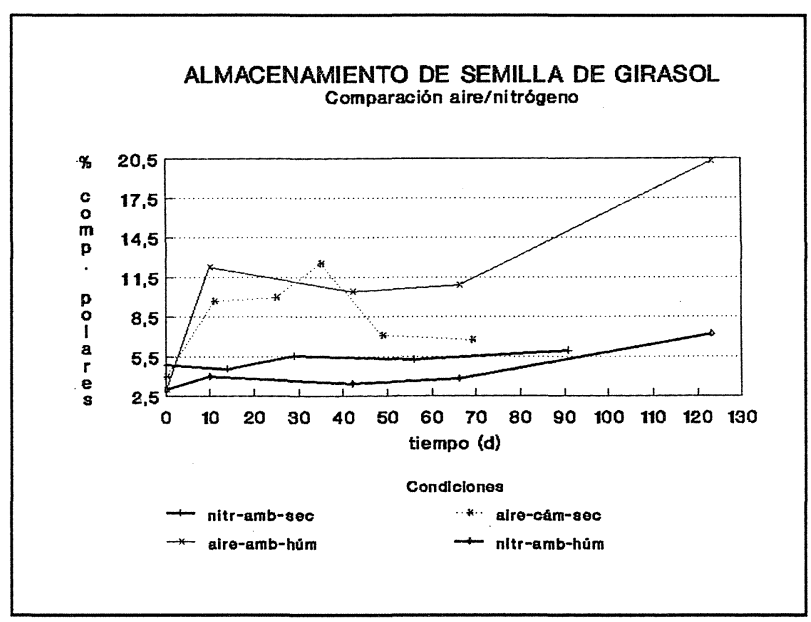

Gráfica 6

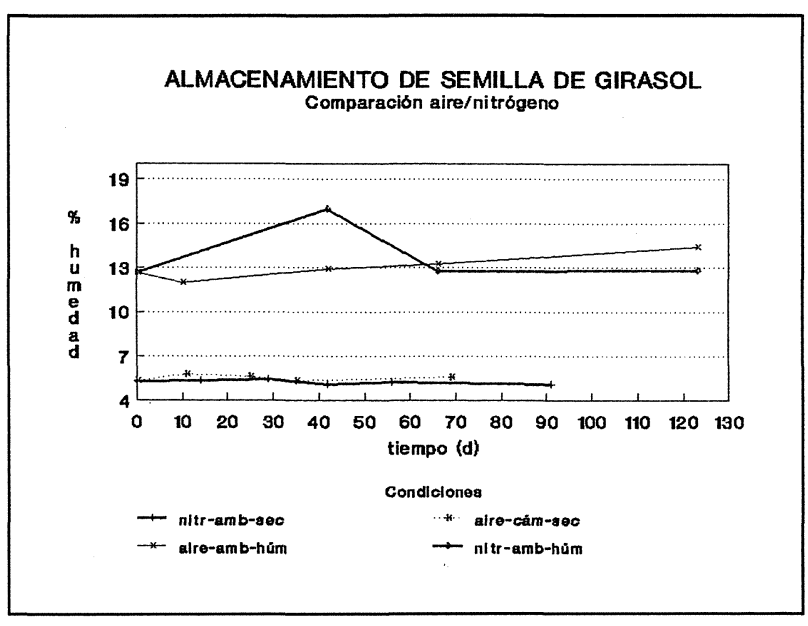

Gráfica 8

humedad conservada en corriente de nitrógeno, se puede decir que en general, en semilla seca existe un buen mantenimiento de la humedad en valores constantes, aunque mejor en nitrógeno que en aire, y en semilla húmeda este efecto se ve potenciado. Esto se puede explicar porque en el caso de la semilla con alta humedad se produce un equilibrio en el aire intergranular de la semilla, pudiendo observar rápidamente la formación de mohos, además de la aparición de la plaga del gorgojo. Estos son otros dos factores que pueden actuar como potenciadores del aumento de acidez de esta semilla.

\section{CONCLUSIONES}

- La semilla de girasol debe ser almacenada en un nivel óptimo de humedad, pero si el almacenamiento debe realizarse con semilla húmeda o en lugares donde no sea posible realizar el proceso de secado, puede retardarse la alteración usando corriente de nitrógeno. 
- La presencia de nitrógeno parece retardar todos los procesos de alteración de la semilla: aumento del grado de acidez, incremento del contenido en compuestos polares, índice de $p$-anisidina, y coeficientes de absorción en el UV a 232 y $270 \mathrm{~nm}$, es decir, retrasa la evolución de compuestos hidrolíticos, térmicos y oxidativos.

- El efecto anterior se ve potenciado al aumentar el contenido en humedad de la semilla.

- Los costes económicos debidos a las pérdidas de aceite crudo por aumento de acidez son muy importantes en todos los casos, pudiendo justificar el uso de nitrógeno a nivel industrial, al menos a nivel experimental hasta la realización de las correspondientes pruebas industriales de almacenamiento de semilla incluyendo la optimización de flujos de inerte.

\section{AGRADECIMIENTOS}

Agradecemos al CICYT por el proyecto ALI-0999-91 concedido, así como al Ministerio de Educación y Ciencia por la beca OPI concedida para la realización del presente trabajo.

\section{BIBLIOGRAFIA}

1.- Eguiazu, G.M.; Motta, A. (1986).- "Relación entre contenido de agua y materia grasa en muestras comerciales de girasol para una humedad relativa ambiente del aire intergranular del 75\%".- Grasas y Aceites 37, 307-312.

2.- Eguiazu, G.M.; Motta, A.- "Intergranular relative air humidity as a parameter of biological deterioration in stored sunflower", en XI International Sunflower Conference.

3 .- Robertson, J.A.; Roberts, R.G.; Chapman, G.W. (1985).- "Changes in oil-type sunflowerseed stored at $20^{\circ} \mathrm{C}$ at three moisture levels".- J. Am. Oil Chem. Soc. 62, 1335-1339.
4 .- Eguiazu, G. M. (1984).- "Comportamiento de almacenaje del girasol. I. Comportamiento de absorción de humedad atmosférica del aquenio, pericarpio y semilla del grano de girasol".- Grasas y Aceites 35, 246250.

5.- Eguiazu, G.M.; Grünewald, Th. (1984).- "Comportamiento de almacenaje del girasol. Il. Cinética de la absorción para muestras de variado origen".- Grasas y Aceites 35, 320-324.

6.- Eguiazu, G.M. (1984).- "Comportamiento de almacenaje del girasol. III. Aparición del primer micelio visible como primer síntoma de deterioro en función de la humedad crítica de la muestra, la humedad relativa ambiente y el tiempo de almacenaje".- Grasas y Aceites 35, 325-329.

7.- Motta, A.; Eguiazu, G.M. (1991).- "Variación de parámetros de calidad del grano y aceite de girasol almacenado a diferentes humedades relativas".- Grasas y Aceites 42, 371-375.

8.- Eguiazu, G.M.; Frank, H.K. (1984).- "Counts of fungal propagules and acid number in sunflower seeds".- Fette, Seifen, Anstrichmittel 86, 16-18.

9.- Robertson, J.A.; Chapman, G.W.; Wilson Jr, R.L. (1984).- "Effect of moisture content of oil type sunflower seed of fungal growth and seed quality during storage".- J. Am. Oil Chem. Soc. 61, 768-771.

10.- Turkulov, J.; Veselinovic, S.; Dimic, E. (1989).- "Contribution to the investigation of chemical changes in sunflowerseed during storage".Fett. Wiss. Technol. 91, 391-393.

11.- Shejbal, J.(Publ. 1979).- "Preservation of cereals and oilseeds in a nitrogen atmosphere".- Dif. Antiparassit. Ind. Aliment. Prot. Alimenti, Atti Simp, 2nd 1977, 185-200.

12.- Tanteeratarm, K.; Wei, L.S.; Steinberg; M.P. (1989).- "Effect of soybean madurity on storage stability and process quality".- J. Food Sci. 54, 593597.

13.- AOCS Official Method Ai 2-75.

14.- Norma UNE 55.011-73

15.- Norma UNE 55.024-73

16.- Norma UNE 55.127-81

17.- Waltking, A.E. y Wessels, H. (1981).- "Chromatographic separation of polar and nonpolar components of frying fats".- J. Assoc. Anal. Chem. 64, 1329.

18.- Patterson, H.B.W.- "Handling and storage of oilseeds, oils, fats and meals", Ed. Elsevier Applied Science, Londres (1989), pg 309.

Recibido: Noviembre 1994

Aceptado: Marzo 1995 